\title{
Linear and Nonlinear Reconstruction Algorithms for Atomic-Resolution Tomography Using Phase Contrast Electron Microscopy
}

\author{
Colin Ophus ${ }^{1}$, David Ren ${ }^{2}$, Michael Chen ${ }^{2}$, Catherine Groschner ${ }^{3}$, Mary Scott ${ }^{1,3}$, and Laura Waller \\ 1. Molecular Foundry, Lawrence Berkeley National Laboratory, Berkeley, USA. \\ 2. Department of Electrical Engineering and Computer Science, UC Berkeley, Berkeley, CA \\ 3. Materials Science and Engineering, UC Berkeley, Berkeley, CA
}

Electron tomography has had an incredible impact on both biological and materials science. In biological sciences, cryogenic electron microscopy (cryo-EM) coherent imaging methods have solved the structure of an enormous number of protein structures, made possible because thousands of identical copies of each molecule can be produced [1]. In materials science, the 3D atomic structure of individual unique nanoparticles can now be determined using an incoherent imaging mode of scanning transmission electron microscopy (STEM) [2], and recently including even identification of chemical species at the atomic level [3] - an emerging field known as atomic electron tomography (AET). These studies were enabled by the ability of metal nanoparticles to handle the large electron dose required for atomic resolution imaging at many orientations. However, neither AET nor cryo-EM (as they currently exist) can solve the 3D atomic structure of unique, low atomic number or dose sensitive nanoscale structures such as polymers, oxide catalysts, or materials containing lithium.

To extend AET to such samples will require using a highly efficient imaging modality, specifically phase contrast methods such as focal series high resolution transmission electron microscopy (HRTEM), ptychography, the recently developed MIDI-STEM method [4], or some other even more dose efficient method such as quantum metrology [5]. These methods extract significantly more information per electron but can produce extremely complex phase contrast patterns. This is because at atomic resolution, the signal oscillates wildly across atomic groups or columns, with a large degree of phase wrapping possible. Practical experiments will require state-of-the art alignment and reconstruction methods [3, 6, 7], algorithms to solve for and remove residual coherent wave aberrations, and the highest quality aberration-corrected HRTEM imaging. In this talk, we demonstrate a two new algorithmic frameworks to reconstruct 3D atomic structure from many different projection angles of low signal-to-noise phase contrast images.

Figure 1a and b shows the experiment geometry we consider: a needle sample tilted around the extended axis of the needle. We have successfully used this geometry for previous AET experiments [2]. HRTEM images simulated using the methods and potentials described in Kirkland [8] are shown in Figure 1c. These same images with Poisson noise for a low dose measurement are shown in Figure 1d. Slices of the 3D atomic potential are shown in Figure 1e.

Our first phase contrast AET reconstruction algorithm couples amplitude-based update steps for complex wave reconstruction [9] with a standard simultaneous iterative reconstruction technique (SIRT) reconstruction. By alternating between 2D and 3D update steps, we can produce a fairly accurate reconstruction, shown in Figure 1f. Almost every atomic site produces a local maximum in the reconstructed volume, demonstrating atomic-resolution contrast. However, substantial artifacts are present, both inside and outside of the reconstructed volume. These artifacts are due to the strong dynamical contrast that is produced when the sample is aligned along low-index crystallographic 
directions. To address these shortcomings, we have also implement a full non-linear inverse multi-slice algorithm, similar to those proposed in $[6,7]$. By including multiple scattering and using a fast gradient descent algorithm, we achieve a significantly more accurate reconstruction, which is shown in Figure $1 \mathrm{~g}$ [10]. In this talk, we will outline both methods and show atomic fitting results for both. The dependence of position error, species identification accuracy and number of false positives on the sample thickness, scattering cross section, electron dose and experimental parameters will be explored in detail [11].

\section{References:}

[1] E Nogales, Nature Methods 13 (2016), p. 24-27.

[2] R Xu et al., Nature Materials 14 (2015), p. 1099-1103.

[3] Y Yang et al., Nature 542 (2017), 75.

[4] C Ophus et al., Nature Communications 7 (2016), p. 10719.

[5] T Juffman et al., Scientific Reports 7 (2017), p. 1699.

[6] W Van den Broek and C Koch, Physical Review B 87 (2013), p. 184108.

[7] L Tian and L Waller, Optica 2 (2015), p. 104.

[8] E Kirkland, Adv. Computing in Electron Microscopy, Springer Science \& Business Media (2010).

[9] L-H Yeh et al., Optics Expresss 23 (2015), p. 33214.

[10] A beck and M Teboulle, SIAM Journal on Imaging Sciences 2 (2009), p. 183.

[11] Work at the Molecular Foundry was supported by the Office of Science, Office of Basic Energy Sciences, of the U.S. Department of Energy under Contract No. DE-AC02-05CH11231.

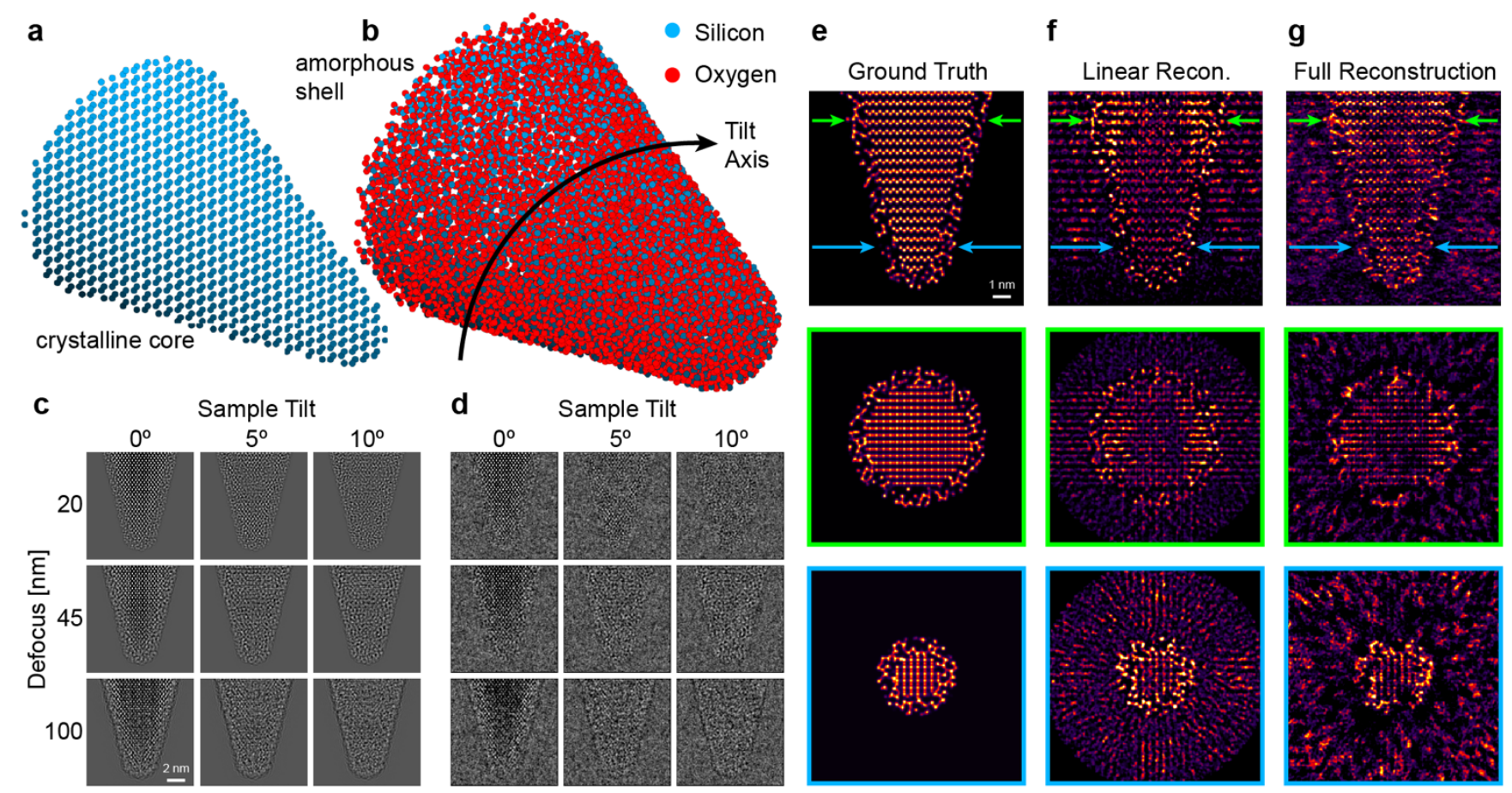

Figure 1. Simulated atomic electron tomography using 3 defocus planes per tilt, 36 tilts total and a dose of 92 electrons / Angstrom ${ }^{2}$ in each image. Atomic coordinates of the sample, showing (a) crystalline core and (b) amorphous shell. (c) Some electron wavefunctions after propagation through sample, corresponding to (d) intensity measurements. (e) Slice of the original 3D potential taken from the middle of the sample, with cross-section slices shown below. (f) Slices of the 3D potential reconstructed with the linear algorithm using the images shown in (d). (g) Slices of the 3D potential reconstructed with the full non-linear reconstruction algorithm using the images shown in (d). 